\title{
Impacto en la salud pública colombiana de los departamentos de medicina preventiva y salud pública de las facultades de medicina
}

\author{
Guillermo Restrepo Chavarriaga ${ }^{1}$, Juan Carlos González Quiñonez²; Martha Isabel Sarmiento Osorio ${ }^{3}$
}

Grupo de Investigación en Atención Primaria, Modelos de Salud y Educación Médica. En la línea de Educación Médica.

1 Médico, Magister en Salud Pública. Director Departamento de Planeación. Docente, Fundación Universitaria Juan N. Corpas.

2 MD. Especialista Medicina Integral Familiar. Magister en Salud Pública. Director Departamento Medicina Comunitaria. Docente, Facultad de Medicina, Fundación Universitaria Juan N. Corpas.

3 Comunicadora Social y Periodista. Coordinadora Departamento de Planeación, Fundación Universitaria Juan N. Corpas.

Correspondencia:

Dr. Guillermo Restrepo Chavarriaga, guillermo.restrepo@juanncorpas.edu.co

Como citar: Restrepo G, González JC, Sarmiento MI. Impacto en la salud pública colombiana de los departamentos de medicina preventiva y salud pública de las facultades de medicina. Cuarzo 2015; 21 (2): 68-73.

Recibido: 15 de junio de 2015.

Aceptado: 30 de agosto de 2015.

Publicado: 15 de diciembre de 2015.

Licencia creative commons

\section{Resumen}

En este artículo se exponen los resultados de una investigación que tuvo por objetivo presentar las diversas orientaciones de los departamentos de medicina preventiva en las décadas del 50 y 60 y su influencia en la educación superior colombiana. La metodología consistió en un estudio histórico basado en un análisis a partir del concepto de medicina integral. Entre sus resultados se destaca que si bien es cierto que Abraham Flexner le daba mucha importancia a los aspectos sociales de la medicina, en la implantación de su modelo en los EE.UU se siguió un enfoque fundamentalmente biologista, sin embargo en Colombia al introducir la modernización preconizada por él, la cual siguió el ejemplo de las Facultades de Medicina norteamericanas, en las que se formaron nuestros líderes en este campo, los Departamentos de Medicina Preventiva fueron un distintivo fundamental. Hecho que significó una gran modificación del modelo. Con la investigación se pudo concluir que tres Facultades de Medicina crearon modelos de desarrollo específicos para estas reparticiones, los cuales por servir de patrones, tuvieron un gran impacto en la educación médica colombiana primero, en la universidad como un todo después, en el Sistema Nacional de Salud posteriormente y a nivel internacional fueron ejemplo no solo en Latinoamérica sino para muchos otros países del mundo.

PALABRAS CLAVE: medicina social, modelo biopsicosocial, historia de la medicina, medicina familiar, educación médica.

\section{Abstract \\ Impact on the colombian public health departments of pre- ventive medicine and public health medical schools}

This article presents the results of an investigation that aimed to present the various orientations of the Department of Preventive Medicine in the decades of 50 and 60 and their influence on Colombian higher education are discussed. The methodology consisted of 
a historical study based on an analysis from the concept of integrative medicine. Among its results emerged that while it is true that Abraham Flexner gave much importance to the social aspects of medicine, in the implementation of the model in the US a fundamentally biologista approach was followed, however in Colombia to introduce modernization advocated by him, which followed the example of the American Schools of Medicine, where our leaders were trained in this field, the Departments of Preventive Medicine were a fundamental distinction. This fact meant a modification of the model. Through research it was concluded that three medical schools created models specific development for these departments, which serve pattern, had a great impact on the Colombian medical education first at the university as a whole later in the National System Health and internationally were subsequently example not only in Latin America but for many other countries.

KEYWORDS: Social medicine, biopsychosocial model, history of medicine, family medicine, medical education..

\section{Introduction}

Cuatro etapas se han presentado en la enseñanza de la medicina preventiva y la salud pública en Colombia las cuales se hicieron más evidentes a partir de la década de los 40. Cada una de estas etapas ha tenido diferente impacto a nivel tanto de la educación médica como en las universidades, en el sistema de salud y aun internacionalmente, según haya logrado cada una de ellas ser innovadora en sus aportes.

\section{Material y métodos}

1. Se entrevistaron los actores que participaron en la realización del programa en alguna de sus fases, en donde relataban la labor ejecutada por ellos en el mismos, apreciación general de este y los resultados obtenidos.

2. Se hicieron visitas a las Facultades de Medicina respectivas, con el fin de buscar testigos que vivieron el proceso o que se enteraron de la aplicación del mismo.

3. Se consultó bibliografía de la época, cuyos autores fueron participantes o testigos del proceso.

4. Se consultó bibliografía general que nos permitiera dar un enfoque global de los conceptos que implica la puesta en marcha de este proceso.

5. Se realizaron entrevistas a personas que dirigieron alguno de estos programas.

6. Se obtuvo en la Biblioteca de la Facultad de Medicina información bibliográfica de la época. Obtenida la información se compacto en orden cronológico se hizo análisis de la misma por el grupo de investigación y se presenta en este documento.

\section{Discusión}

El análisis de la información determinó la existencia de cuatro etapas en la enseñanza de la medicina preventiva y la salud pública.

\section{Primera etapa: la higiene}

Hasta finales del decenio de los $40 \mathrm{y}$ desde que se estructuró un verdadero currículo en las escuelas de medicina con el modelo de francés, propuesto por Mutis (1) la enseñanza de lo que hoy se llama medicina preventiva y salud pública, se concretaba fundamentalmente en lo que en su momento tanto nacional como internacionalmente era: la higiene.

Este tipo de enseñanza obedecía primordialmente a un pensamiento derivado de la Ilustración francesa, (2) en la que se proponía un estado liberal con grandes libertades individuales y poca intervención en las actividades privadas. Las características más importantes de esta etapa fueron las siguientes:

\section{Estructura académica}

No era departamentalizada (3) y a modo de cátedras se enseñaban aspectos de aseo personal, vigilancia y control en inmuebles y establecimientos públicos, algunas vacunas y nociones de bioestadística fundamentalmente descriptiva. En algunas Facultades este trabajo se acompañaba de visitas a establecimientos como mataderos (4). Esta enseñanza se realizaba generalmente así: La parte de bioestadística, en una hora o dos semanales fundamentalmente en los primeros años de la carrera y la higiene propiamente dicha en los últimos años de la misma. 


\section{Docentes}

Los profesores de higiene eran por lo general médicos funcionarios públicos, la mayoría de ellos de gran prestigio nacional, social y político y su vinculación era por concurso y con una dedicación de tiempo parcial, solamente el correspondiente a las horas de clase que debían dictar. La parte de bioestadística se dictaba como un segmento independiente sin ninguna vinculación con lo correspondiente a la higiene.

\section{Estudiantes}

La enseñanza de estas materias era considerada por el estudiantado como elementos accesorios a su formación profesional y por lo tanto vista en un plano muy secundario (5)

Segunda etapa: la medicina preventiva y la salud pública

Con el cambio de sitio de especialización de nuestros profesionales médicos en el exterior y como consecuencia de la última Guerra Mundial, la corriente de médicos que buscaban profundizar en sus conocimientos, cambió de Europa y sobre todo de Francia hacia los EE.UU.

Para esta época se había producido en Norteamérica un cambio rotundo en la formación Médica motivado por la propuesta que hizo Abraham Flexner (6) para mejorar la calidad de esta carrera en las Facultades de Medicina de EE.UU. y Canadá.

Los médicos que regresaban, bien fuera como docentes o en ambientes profesionales por fuera de la Universidad, como eran las academias de medicina y las revistas médicas que circulaban en el país, empezaron a publicar sobre los aspectos que ellos habían vivido en su especialización en relación con los avances que se estaban dando en los países del norte.

Algunos de estos profesionales vinculados a las Facultades en cargos directivos, empezaron hacer reformas importantes en la educación médica colombiana en las tres Facultades de medicina existentes a finales de la década de los 40. Queremos destacar las modificaciones introducidas en la Universidad de Antioquia (7) sobre selección de estudiantes para ingreso, prohibición de repeticiones indefinidas de las materias y vigorización de las ciencias básicas, con la vinculación de profesores extranjeros de tiempo completo. Estas modificaciones produjeron en esta Universidad, una huelga importante que, afortunadamente, no logró modificar las transformaciones que se habían implementado.

Tres Escuelas de medicina fueron pioneras en el cambio ya en una forma integral, estas Escuelas fueron la de la Universidad de Antioquia, la Facultad de Medicina de la Universidad del Valle recientemente fundada y la Universidad de Cartagena.

Para comienzos de 1950 la Facultad de Medicina de la Universidad de Antioquia con el liderazgo de su decano, introdujo una serie de modificaciones en esta entidad de tipo flexneriano, pero con un énfasis especial que modificaba sustancialmente el carácter biologista que implicaba la implantación de ese modelo.

Este cambio lo constituyó la creación del Departamento de Medicina Preventiva (1954) (8-11) que, con un carácter eminentemente social, llevaba a ejecutar un modelo biopsicosocial e histórico (12) que ejerció gran impacto en la educación médica primero, en las ciencias de la salud, posteriormente, y en el sistema de salud después.

Para ejecutar estas labores designo un profesional, que además de salubrista tenía una amplia visión de la medicina social.

Cuatro programas se ejecutaron que caracterizaron estas transformaciones:

A. El desarrollo de la cátedra (13): Se realizaba como un bloque en el cuarto año de Medicina el cual incluía los aspectos de:

- Filosofía de la salud pública.

- Los factores sociales determinantes (14) de la situación de la salud principalmente:

- Alcoholismo y drogadicción.

- Diversos tipos de violencia.

- Prostitución.

- Aspectos económicos y salud.

- La organización sanitaria colombiana. 
- Discusión en mesas redondas sobre lo encontrado en las familias.

B. El Programa Familiar (15): Realizado tanto en algunos barrios de Medellín a los cuales servían Centros de Salud del Municipio y en una zona rural. Este programa consideraba los factores sociales y culturales como componentes etiológicos en la intervención del proceso salud y enfermedad en las personas, la familia y la comunidad.

C. El Programa Rural: Desarrollado en una población Antioqueña (16) en donde además de ejecutar en él, el programa familiar se estableció un modelo de organización sanitaria que contempló todos los aspectos de lo que muy posteriormente en 1978, denominó la OMS atención primaria, a raíz de la reunión de Alma Atta. Este modelo se montó con base en una estructura comunitaria, que cubría la zona urbana del municipio y sus veintidós veredas, con juntas de salud, que realizaban una labor intersectorial y capacitando innovadoras denominadas promotoras de salud que ejercían una función de medicina simplificada, registros demográficos básicos en su comunidad, seguimiento de hechos vitales en las mismas y una amplia labor en promoción de la salud, prevención de la enfermedad y educación sanitaria, con extensión así como labore de vacunación y saneamiento ambiental,

D.Por último, el logro mayor del departamento fue la creación de la Escuela Nacional de Salud Pública (17) que, aunque inicialmente estaba vinculada a éste, gozaba de gran independencia. En esta Escuela se desarrolló el primer programa de magister para médicos, enfermeras y educadores en Salud, en Colombia. En ella se refleja con toda claridad la filosofía biopsicosocial del Departamento, la cual pretendía formar el equipo de salud proyectado a la comunidad.

El otro programa desarrollado fue el que estructuró la Universidad del Valle (1956) en su Facultad de Medicina en donde gracias al liderazgo que su Director logró, en toda la comunidad docente de esa entidad, y con el aporte y estrecha colaboración de la Fundación Rockefeller, se estableció un modelo que elevó al más amplio nivel científico nuestra educación médica.

También cuatro hechos se destacaron en este programa que fueron los siguientes:

A. La estructuración curricular del Departamento se hizo a lo largo de toda la carrera, teniendo como espina dorsal el Programa Familiar y sirviendo de hilo conduc- tor, que integraba verticalmente el Programa de Medicina y con gran penetración transversal por la colaboración que otros Departamentos le hacían. También se logró una colaboración transdisiplinaria sobre todo con las Facultades de Arquitectura e Ingeniería Sanitaria.

B. El programa familiar realizado en un barrio de Cali, en donde se hacían estudios epidemiológicos de la forma como se produce la trasmisión y propagación de la enfermedad en un grupo primario, así como la importancia de la colaboración interdisciplinaria primero, con otras especialidades médicas y trasdiciplinaria con otras Facultades de la Universidad en la compresión y solución de los problemas médicos en un área.

C. El otro eje fundamental del desarrollo de ese departamento, fue la investigación no solo en el programa familiar antes mencionado sino en diferentes trabajos de profundización en varios campos, con participación de los distintos departamentos de la Facultad y de entidades nacionales e internacionales. Cabe anotar que este departamento desarrollo otros tipos de programas tanto urbanos como rurales, sobre todo en un municipio cercano a Cali, llamado Candelaria (18)

D. Este Departamento en 1968 empezó a formar Magister en Salud Publica (19) para médicos, con una gran formación científica y un enfoque epidemiológico.

Posterior a este desarrollo independiente, de cada uno de los departamentos, por las frecuentes reuniones que había entre los docentes de las distintas Facultades, estos programas se retroalimentaron mutuamente (20).

Finalmente, la Universidad de Cartagena en el Departamento de Medicina Preventiva (1960) en su Facultad de Medicina, gestó un cambio que siguió en general los patrones del Valle, pero introdujo dos modificaciones de gran trascendencia como fueron:

A. La creación de un departamento que se encargó de la enseñanza de los aspectos de la medicina preventiva y salud pública para toda la Universidad.

B. La extensión hacia el servicio de salud tomando como su responsabilidad la organización sanitaria de la ciudad de Cartagena, a todos los niveles del sistema.

También es importante anotar que su gestor había sido formado en la Escuela de Salud Pública de Chile (21) en 
donde se seguía ya el patrón norteamericano y con algunos elementos de las propuestas de medicina social europeas.

Tercera etapa: la contrarreforma y la diáspora

En los decenios del 70 y del 80 (22-24), se produjo un movimiento de contrarreforma que trataba de suprimir la intervención norteamericana en la educación médica de nuestro país. La financiación que entidades estadounidenses daban a nuestras Facultades en diversas formas, fue duramente cuestionada sobre todo por los programas, materno infantiles que implicaban un componente de planificación familiar, que estas habían implementado. De la misma manera los centros de práctica comunitarios se consideraron como laboratorios de experimentación en nuestras poblaciones.

Como hecho importante con relación a este gran movimiento nacional, el currículo como tal no fue modificado, pero si sus ejecutores, directivos y sobre todo la financiación que sustentaba su ejecución, bajando la calidad de los mismos, puesto que la planeación de lo que se ejecutaba fue modificada por la improvisación. Como consecuencia importante de todo lo anterior se produjo:

A. Una expulsión de directivos y docentes de alta calidad y su remplazo por profesores improvisados a nivel de las Facultades en general.

B. Disminución de la investigación.

C. Finalización de los campos de práctica universitarios, extrahospitalarios.

D. Supresión de las publicaciones científicas.

Como consecuencia de este gran movimiento estudiantil y de algunos de los docentes, se produjo una diáspora de profesores expulsados y de estudiantes que empezaron a buscar su formación médica en entidades que les pudieran garantizar menos improvisación y sobre todo mayor seguridad en el cumplimiento de lo programado.

De las dos filosofías que se habían implementado, la biopsicosocial histórica y la biopsicologica, la última se propagó más, bien sea en forma total o parcial en las nuevas Facultades creadas y así en el momento actual, podemos hacer un resumen de la enseñanza de la medicina preventiva y salud pública de la siguiente manera que consideraremos como una cuarta etapa:

\section{Cuarta etapa: programas a granel}

Si alguna característica tiene en este momento los programas de medicina preventiva y salud pública, es su gran heterogeneidad (25). De la gran importancia que tuvieron en la década del 50 y 60 los departamentos de medicina preventiva pasaron a diluirse en la mayoría de los nuevos programas en las estructuras académicas de los programas de medicina.

Un breve resumen de lo que en este momento podemos encontrar, lo pasamos a enunciar a continuación:

\section{Estructura académica}

No es uniforme (26), el número de créditos que cada Facultad dicta es distinto, en algunas de ellas no existe Departamento debidamente estructurado de estas disciplinas, el contenido de las unidades, sus denominaciones, la duración y la ubicación es variable, algunos de sus temas prácticos se consideran como electivos y solo de obligatoriedad para algunos estudiantes que como contribución social deben hacer a las becas que les otorgan, no hay ni siquiera acuerdo entre los nombres de las unidades y del lenguaje que se utiliza para designarlas(27). Tal vez el único elemento que es constante es la epidemiologia.

\section{Docentes}

La mayoría de ellos son magister en salud pública o epidemiologia y algunos son PHD, de dedicación variable a la Facultad. Sin embargo, los relacionados con las ciencias sociales, no tienen frecuentemente conexión con los salubristas, lo propio sucede con la bioestadística que en la mayoría de escuelas tiene un contenido más orientado a una estadística general descriptiva. La administración sanitaria tiene connotaciones diferentes en los distintos programas.

\section{Estudiantes}

Fuera de la epidemiología, no ven en este departamento una masa de conocimientos universalmente concebidos que los caracterice. Frecuentemente desde los primeros años de la carrera ya tienen definida su especialidad, la cual casi en su totalidad está orientada a las áreas clínicas.

En estas condiciones la mayoría de la estructura de nuestras Facultades sigue un esquema biologista y aunque se han incorporado en algunos de ellos las ciencias 
sociales estas se miran solamente como un componente humanístico sin una debida integración con los problemas de salud de las personas, las familias y sus comunidades.

La estadística pertenece más a las ciencias exactas y la administración si se da, tiene más un enfoque gerencial que de modelo de prestación de servicios de salud.

Finalmente un hecho grave para la salud pública colombiana fue que desapareció la comunidad de salubristas (28) que proponían, las líneas a seguir en estos aspectos no solo en la academia sino en la salud pública en general.

\section{Referencias}

1. Cortes García Claudia M. Historia de la medicina en Colombia. De la medicina ilustrada a la medicina anatomoclínica, 1782-1867 - Tomo II. En: Revista de Gerencia y Políticas de Salud. Vol. 9. No. 18. Bogotá. Junio. 2010

2. Ibíd., Vol. 9. No. 18.

3. Informe del Dr. Ocampo Londoño. Memoria del Ministro de Salud Pública al Congreso Nacional. República de Colombia-Ministerio de Salud Pública. Bogotá D.C. 1960.

4. Vélez Escobar Ignacio. Una experiencia maravillosa. La Fundación del Café. Libro Historia de la Nueva Universidad de Antioquia (segunda parte) y otros temas. Pp. 41-45.

5. Flexner Abraham. (1866-1959). The American College: A Criticism, New York, The century Co., 1908.

6. Vélez Escobar Ignacio. Libro, La Facultad de Medicina de la Universidad de Antioquia 1935-1965. Pp. 47-48.

7. Conferencia Enseñanza de la Medicina Preventiva. Colorado Springs. Estados Unidos. 1952.

8. Dr. Pazos Varela Ricardo. Presidente., Dr. Krumdieck Carlos F., Trelles J.O. Secretarios Generales. I Congreso Panamericano de Educación Médica. Organizado por la Facultad de Medicina de la Universidad de San Marcos. Lima, Perú. Acta Final. Mayo 14-18, 1951.

9. Seminario Sobre la Enseñanza de Medicina Preventiva. Oficina Sanitaria Panamericana. Oficina Regional de la Organización Mundial de la Salud Washington, D.C. Viña del Mar, Chile 10-15 de octubre, 1955. Tehuacán, Mexico23-28. Abril. Pp. 121956.
10. Seminarios Sobre la Enseñanza de Medicina Preventiva. Oficina Sanitaria Panamericana. Oficina Regional de la Organización Mundial de la Salud Washington, D.C. Viña del Mar, Chile 10-15 de octubre, 1955. Tehuacán, Mexico23-28. Abril. Pp. 121956.

11. Vélez Escobar Ignacio. Libro la Facultad de Medicina de la Universidad de Antioquia 1935-1965.Pp 47-48.

12. Los Determinantes Sociales de la Salud. Ministerio de Sanidad y Consumo. Dirección General de Salud Pública. OMS. España.

13. Vélez Escobar Ignacio. Libro La Facultad de Medicina de la Universidad de Antioquia 1935-1965. Pp. 51-52

14. Libro Modernización de la Educación Medica Colombiana a Partir de la Influencia Germano-Norteamericana. 1940-2015. Capitulo IV. Facultad Nacional de Salud Pública y Escuela de Salud Pública.

15. Llanos G., Pradilla A., Rueda A. Libro. Candelaria El Principio de la Medicina Social. Edición: Fundación Éxito. Impresión: Marquilla S.A. Medellín. Octubre de 2007.

16. Henao Cabal Oscar., M.D., M.P.H. Serie: Lecturas Escogidas. Crónica de la Escuela de Salud Pública de la Universidad del Valle. Publicación Científica No. 13.Escuela de Salud Pública Facultad de Salud. Cali, Colombia. 1996.

17. Comunicación personal del Dr. Abel Dueñas Padrón.

18. Revista. Historia de la Educación Latinoamericana. Universidad Pedagógica y Tecnológica de Colombia. ISSN (Versión Impresa): 0122-7238.Tunja. Colombia. 2005.

19. Henao Cabal Oscar., M.D., M.P.H. Serie: Lecturas Escogidas. Crónica de la Escuela de Salud Pública de la Universidad del Valle. Publicación Científica No. 13.Escuela de Salud Pública Facultad de Salud. Cali, Colombia. 1996.

20. Vélez Escobar Ignacio. Libro La Facultad de Medicina de la Universidad de Antioquia. 1935-1965.

21. Dr. Restrepo Ch. Guillermo., Dra. Iregui., Dr. Bustillo José., Dra. Quesada Beselink., Dra. Perdomo Victoria. Análisis de la Primera Fase Análisis de Pensum de Medicina Universidades Colombianas. 2010.

22. Dr. Restrepo Ch. Guillermo., Dra. Iregui., Dr. Bustillo José., Dra. Quesada Beselink., Dra. Perdomo Victoria. Análisis de la Primera Fase Análisis de Pensum de medicina Universidades Colombianas. 2010.

23. Informe personal del Dr. Germán Ortiz. Ex. Presidente de la Academia Colombiana de Salud 